\section{PSICOLOGIA IBEROAMERICANA}

\section{Psicología lberoamericana}

ISSN: 1405-0943

psicología.iberoamericana@uia.mx

Universidad Iberoamericana, Ciudad de

México

México

Garnica Jaliffe, Gustavo; Robles García, Rebeca; Sánchez Sosa, Juan José; Juárez García, Francisco Mejoramiento de contingencias parentales y reducción de problemas conductuales en adolescentes

Psicología Iberoamericana, vol. 21, núm. 1, enero-junio, 2013, pp. 79-87

Universidad Iberoamericana, Ciudad de México

Distrito Federal, México

Disponible en: http://www.redalyc.org/articulo.oa?id=133929862009

Cómo citar el artículo

Número completo

- Más información del artículo

- Página de la revista en redalyc.org

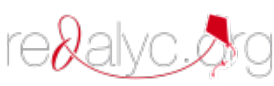

Sistema de Información Científica

Red de Revistas Científicas de América Latina, el Caribe, España y Portugal Proyecto académico sin fines de lucro, desarrollado bajo la iniciativa de acceso abierto 


\title{
Mejoramiento de contingencias parentales y reducción de problemas conductuales en adolescentes
}

\author{
Parental Contingencies Improvement and Behavioral Problems \\ Reduction in Adolescents
}

\author{
Gustavo Garnica Jaliffe* \\ Facultad de Psicología, unam \\ Rebeca Robles García \\ instituto Nacional de Psiquiatría \\ Juan José Sánchez Sosa \\ Facultad de Psicología, unam \\ Francisco Juárez García \\ instituto Nacional de Psiquiatría
}

\section{RESUMEN}

Debido a su importancia para prevenir conductas de riesgo, se describieron los problemas conductuales de 17 adolescentes con una edad promedio de 14 años con sus padres, así como las afectaciones percibidas por ambas partes. Se intervino mediante un diseño $\mathrm{N}=1$ pre/post test y un modelo conductual para aumentar la incidencia de conductas adecuadas y reducir la de inadecuadas. Los resultados mostraron la efectividad en la modificación conductual y en la percepción de afectaciones tanto en padres como en adolescentes. Se comentan ciertas limitaciones.

Palabras clave: adolescentes, padres, manejo de contingencias, conductas problema.

\section{ABSTRACT}

We analyzed the problems between 14 years old adolescents and their parents, the damages received by them and treated by a $N=1$, pre-post test design, and a behavioral model to increase appropriate behaviors and reduce inappropriate ones, due to its importance in preventing risk behaviors. The results showed the effectiveness of behavior modification, the reduction on problems associated with substance use, and the low perception of affectations between parents and adolescents. Limitations are discussed.

Keywords: adolescents, parents, contingency management, problem behavior.

\footnotetext{
* Recibido: 31 de octubre, 2012 - Aceptado: 26 de marzo, 2013

Correspondencia: Gustavo Garnica Jaliffe, gusgajale@hotmail.com

Agradecimientos: Al Consejo Nacional de Ciencia y Tecnología, al Centro Nacional para la Prevención y el Control de las Adicciones, a los Servicios de Salud Pública del Distrito Federal y a la Universidad Iberoamericana Ciudad de México.
} 


\section{INTRODUCCIÓN}

La adolescencia de los hijos puede constituir un reto importante para los padres, quienes con frecuencia requieren de habilidades para manejar adecuadamente la rebeldía, la desobediencia, el descuido de la escuela y los problemas de conducta. Se ha demostrado que de no controlar estos problemas, podrían acarrear otras vicisitudes de mayor relevancia, tales como la deserción escolar, los embarazos a temprana edad, conductas delictivas, el abuso y dependencia a sustancias adictivas, problemas de salud graves o incluso la muerte (Bojórquez, Fernández-Varela, Gorab \& Solís, 2010; Burt, Resnick \& Novick, 1998; Capuzzi \& Gross, 2004; Dryfoos, 1990; Jessor, 1992; Learner \& Spanier, 1978; National Institute on Drug Abuse [NIDA], 2005; Rebolledo, Medina \& Pilon, 2004).

A pesar de que las conductas problemáticas de algunos adolescentes son relativamente cotidianas pero de riesgo potencial, en nuestro país existe poca información acerca de qué comportamientos son problemáticos, con qué frecuencia se presentan, en qué grado afectan a los padres y adolescentes, y de la efectividad de intervenciones psicológicas dirigidas a incrementar las habilidades parentales a fin de manejarlas y reducirlas de manera adecuada.

En otros países se ha documentado que los conflictos característicos de la etapa de la adolescencia incluyen la hora de llegada, el número de veces que el adolescente sale durante las noches, las calificaciones en la escuela, la selección de amistades, el gasto de dinero, modo de vestir, las restricciones para el uso del automóvil, el consumo de alcohol o tabaco, así como la participación en labores domésticas (Papalia, Wendkos \& Duskin, 2007). Esto es señal de que hay problemas en la aplicación de reglas y límites, de que en muchos casos las sanciones no se cumplen y las buenas conductas no son alentadas.

La aplicación de reglas y límites se ha considerado un importante factor de protección (Burt et al., 1998, Capuzzi \& Gross, 2004; Morales, Martínez \& Vázquez, 2007; NIDA, 2005; Villatoro et al., 2007) y su aplicación se puede apoyar en principios de la psicología conductual, específicamente del condicionamiento operante, el cual establece que la correcta aplicación de consecuencias a las conductas individuales permite su modificación (Kazdin, 2000), y que es posible desarrollar o incrementar conductas deseables mediante reforzamiento (Godley, Hedges \& Hunter, 2011) y disminuir o eliminar las inadecuadas mediante castigo (Marini \& Stickle, 2010) o extinción (Rosendahl, Galanti \& Gilljam, 2008).

Los reforzadores positivos más potentes para una persona se encuentran en el comportamiento social, como recibir atención, una caricia, una palabra de aprobación, una sonrisa, etc. (Patterson, 1975). El castigo implica la presentación de un evento aversivo (castigo I) o el retiro de un evento positivo que procede a una respuesta (castigo II), y la extinción se refiere al cese de reforzamiento de una respuesta.

La aplicación de cualquiera de estas contingencias se puede establecer de manera directa: ante una conducta adecuada, aplicar reforzamiento y ante una inadecuada, castigo. Pero también se pueden aplicar contingencias de forma más sofisticada y complementaria, como parte de un proceso de negociación, el cual es una técnica efectiva para la resolución de conflictos interpersonales, así como para el logro del funcionamiento y bienestar social (Fulgencio, 2002; Neale \& Bazerman, 1991) y además ha demostrado ser una técnica efectiva en el control de la conducta del adolescente (Fulgencio, 2002).

Dado que la familia es un factor proximal susceptible de intervención psicológica, la información que se obtenga de ella y el desarrollo de programas de entrenamiento a padres podría contribuir a la solución de conflictos en la adolescencia (Corsi, Barrera, Flores, Perivanicich \& Guerra, 2009; Mahecha, 2006; Morales et al., 2007), la etapa de mayor susceptibilidad a problemas de salud física y/o mental (Millstein et al., 1992).

En esta dirección, el presente trabajo tiene por objetivo describir las problemáticas conductuales que los padres tienen con sus hijos adolescentes, y modificarlas, en base a un entrenamiento a padres en aplicación de contingencias.

\section{MÉTODO}

\section{Participantes}

Los participantes fueron 17 mujeres con edad promedio de 40 años y sus hijos adolescentes (10 hombres y 7 mujeres) de edad promedio 14 años. Acudieron a recibir atención psicológica a un centro comunitario de 
prevención y atención de las adicciones (Centro Nueva Vida) de la Secretaría de Salud del Distrito Federal, que aceptaron participar voluntariamente en el estudio. Se incluyeron a padres que vivían con el adolescente y que no fueran dependientes de alcohol o tabaco ni consumieran sustancias adictivas; así como a adolescentes entre 12 y 17 años sin abuso o dependencia a sustancias, aunque podían presentar consumo experimental.

\section{Variables e instrumentos \\ Conductas adecuadas e inadecuadas \\ de los adolescentes}

En el presente estudio, la conducta adecuada (CA) representa un beneficio para el individuo que la realiza y para los demás, mientras que la conducta inadecuada (CI) significa un perjuicio, tomando en cuenta las definiciones de beneficio/perjuicio del Diccionario Manual de la Lengua Española (2007) y el Diccionario Enciclopédico Larousse (2009). Estas conductas pueden medirse a través de la frecuencia/cantidad de apariciones que se observan en una gráfica de polígonos de frecuencia. Los instrumentos para evaluarlas fueron: a) línea base retrospectiva, un registro del patrón de conductas que se han presentado antes del tratamiento y del primer contacto con la institución que se aplica, con base en un calendario que incluye los cinco días anteriores al contacto con el terapeuta; y b) registro conductual, una tabla de conductas y una gráfica para indicar la cantidad de conductas detectadas por el usuario en un periodo de una semana. Se registra diariamente para identificar tendencias o fluc- tuaciones en el patrón conductual de interés (Cooper, Heron \& Heward, 1987; Kazdin, 2000).

Contingencias erróneas y adecuadas de los padres A las conductas respuesta que tiene el padre ante las CA y las CI se les denomina contingencias, y pueden calificarse también como correctas o erróneas. Las contingencias erróneas serán todas aquellas conductas consecuentes que faciliten el mantenimiento o incremento de la conducta inadecuada y la reducción o extinción de la adecuada. Por su parte, la contingencia correcta es aquella que favorece el aumento de conductas adecuadas y la reducción y/o extinción de las inadecuadas, de acuerdo con los principios del condicionamiento operante (Kazdin, 2000). Además, la conducta respondiente de los padres debe respetar la integridad física y emocional de sus hijos (Fondo de las Naciones Unidas para la Infancia [Unicef], 2006) para ser considerada una contingencia correcta (véase tabla1).

En este trabajo la medición se llevó a cabo mediante el registro de análisis funcional, un registro conductual para observar la interacción entre los antecedentes, la conducta y sus consecuencias. La aplicación consiste en elaborar cadenas conductuales desde el primer antecedente hasta la última consecuencia, aislando una sola conducta por registro. La definición de la conducta es clara y descriptiva, haciendo uso preferencial de verbos en lugar de adjetivos. También se registra la hora, el lugar y las personas que estuvieron presentes ante la aparición de la conducta (tabla 2).

Tabla 1. Errores de reforzamiento de la conducta del adolescente

\begin{tabular}{l|l}
\hline \multicolumn{1}{c|}{ Conducta adecuada } & \multicolumn{1}{c}{ Conducta inadecuada } \\
\hline $\begin{array}{l}\text { Ignorar la conducta (pasar por alto la CA) } \\
\text { Reforzamiento sin valor para el adolescente }\end{array}$ & $\begin{array}{l}\text { Ignorar la conducta (pasar por alto la falta o Cl) } \\
\text { Reforzamiento positivo (intencional o no intencional) }\end{array}$ \\
$\begin{array}{l}\text { Reforzar en exceso } \\
\text { Reforzamiento incoherente con la conducta } \\
\text { Reforzamiento negativo } \\
\text { Castigo }\end{array}$ & $\begin{array}{l}\text { Castigo indeterminado en el tiempo } \\
\text { Castigo desmesurado, no equivalente o incoherente a la Cl } \\
\text { Castigo que incluya maltrato físico, emocional o psicológico }\end{array}$ \\
\hline
\end{tabular}


Tabla 2. Ejemplo de registro de análisis funcional

\begin{tabular}{l|l|l}
\hline \multicolumn{1}{c|}{ Contexto } & \multicolumn{1}{c|}{ Conducta } & \multicolumn{1}{c}{ Consecuencias } \\
\hline $\begin{array}{l}\text { 16:00 hrs. El adolescente está en la } \\
\text { sala chateando por internet. }\end{array}$ & $\begin{array}{l}\text { El adolescente no contesta a su mamá y } \\
\text { sigue chateando en la computadora. }\end{array}$ & Mamá se retira sin decir nada. \\
Mamá le pide hacer su tarea. & & \\
\hline
\end{tabular}

\section{PROCEDIMIENTO}

Se utilizó un diseño de caso único $(\mathrm{AB})$ preexperimental de un grupo pre y postest, y la intervención constó de una sesión de admisión, una de evaluación siete tópicos de intervención. En la sesión de admisión al padre de familia se aplicó una entrevista conductual en donde se exploraron aspectos de la conducta problema, así como percepciones de la magnitud de dichos problemas; posteriormente se le pidió que eligiera cinco conductas adecuadas y cinco inadecuadas, consideradas las conductas blanco a modificar mediante la intervención. En el caso de las adecuadas, se eligieron conductas que el padre deseara que su hijo/a desarrollara o presentara con mayor frecuencia; en el de las inadecuadas, se eligieron las que quisiera eliminar o reducir en su hijo/a.

En la sesión de evaluación se aplicó una línea base retrospectiva a cinco días para contar cada una de las 10 conductas blanco, se realizó el análisis funcional de la conducta de dos conductas adecuadas y de dos inadecuadas para identificar posibles errores de aplicación de contingencias de los padres ante la conducta de sus hijos y se les explicó el llenado del registro conductual.

La intervención comenzó con el tema del diagrama de manejo conductual, mediante el cual el participante se familiariza con toda la intervención y su lógica. En el siguiente tópico se entrenó en el desarrollo o aumento de conductas adecuadas mediante reforzamiento positivo (elogio); para proceder en las siguientes sesiones: las reglas de aplicación de contingencias, la reducción de conductas inadecuadas mediante técnicas de castigo y extinción y finalmente, el aprendizaje de la realización de un análisis funcional y de técnicas de negociación.

\section{RESULTADOS}

La evaluación inicial fue completada por un total de 50 madres de familia y dos matrimonios. Su edad promedio fue de 39 años, con escolaridad secundaria (45\%) y primaria (35.5\%). En cuanto a la descripción y características de los problemas de los padres con sus hijos adolescentes, el 58\% refirió problemas de conduc-

Tabla 3. Conductas blanco seleccionadas por los padres de familia

\begin{tabular}{|c|c|c|c|}
\hline Conductas adecuadas & $\%$ & Conductas inadecuadas & $\%$ \\
\hline Atender la escuela & 14.5 & Mentir & 11.4 \\
\hline Respetar acuerdos & 10 & Desatender la escuela & 11.4 \\
\hline Obedecer órdenes simples & 10 & Mostrarse despreocupado por conductas inadecuadas & 8.6 \\
\hline Realizar actividad provechosa & 10 & Consumir sustancia adictiva & 8.1 \\
\hline Mostrarse preocupado por conductas inadecuadas & 9.5 & Cambios de humor repentinos & 7.6 \\
\hline Interactuar por iniciativa propia & 9.5 & Pasar tiempo de ocio & 7.6 \\
\hline Realizar actividades domésticas & 7.2 & Dormir tarde & 4.8 \\
\hline Mostrar autocontrol & 6.7 & & \\
\hline
\end{tabular}


ta del adolescente en casa, seguidos de problemas de conducta en la escuela (22.6\%), problemas académicos (16.1\%) y de consumo de sustancias (3.2\%). La mayoría de los padres consideraron estos problemas como graves (58\%), indeciso (19.4\%), muy graves (12.9\%) y leves (9.4\%). El 71\% de ellos refirió que su hijo consumía alguna sustancia adictiva. La frecuencia percibida de los problemas fue diario (54.8\%), dos a tres veces por semana (25.8\%), una vez por mes (12.9\%), una vez a la semana (3.2\%) y una vez cada 15 días (3.2\%). La tabla 3 presenta las principales conductas blanco elegidas por los padres de familia.

Finalizaron la intervención 17 padres de familia; en todos se observaron cambios favorables en la aplicación de consecuencias positivas ante conducta adecuada y de consecuencias negativas ante la conducta inadecuada de sus hijos.

En la figura 1 se observa el tipo de contingencia que los padres aplicaron después de la conducta adecuada de sus hijos. Incluye: 1) elogiar, que se refiere a un reconocimiento de la conducta realizada, mirar a los ojos, hacer contacto físico y sonreír (Patterson, 1975); 2) otorgar permisos que van desde ver la tele, salir con amigos, usar la computadora, hasta salir con la pareja o ir a fiestas; 3$)$ interactuar prestando atención, platicando o conviviendo con el adolescente (sea jugando, paseando etc.); 4) recompensa comestible, que es el otorgamiento de algún reforzador comestible como algún postre, platillo o botana; 5) recompensa material, que implica la presentación de algún objeto de valor material como ropa, zapatos, celulares y otros artículos electrónicos o incluso dinero; 6) ayudar en tareas en quehaceres domésticos o escolares como resultado de su buen desempeño conductual; y 7) contingencia errónea, que implicó pasar por alto o no notar una conducta adecuada y por consiguiente, no otorgar ningún reforzador, o a cualquier error de contingencias que pueda eliminar una conducta adecuada, como el castigo I o II.

Como se puede observar, esta última contingencia es muy frecuente al inicio y se extingue al final de la intervención, mientras que con el elogio sucede lo contrario.

En la figura 2 se observa la aplicación de contingencias de los padres ante la conducta inadecuada de sus hijos. Se incluyen: 1) la pérdida de privilegios como estrategia del castigo II del condicionamiento operante (Kazdin, 2000), que se refiere al retiro contingente de reforzadores positivos como permisos, uso de computadora, recompensas materiales, dinero o ir a fiestas, etc; 2) el castigo I del condicionamiento operante, es decir, la presentación de un evento aversivo o molesto para el adolescente para reducir su conducta, por ejemplo, regañar, gritar, pegar, insultar y ponerlo a realizar labores domésticas extras u otro tipo de actividad molesta; 3 ) ignorar, cuando el padre de familia decide intencionalmente retirar la atención a la conducta de su hijo como estrategia ante conductas de su hijo que buscan la confrontación como: gritar, insultar, pegar, o burlarse; 4) advertir, cuando se comunica al adolescente con anticipación la aplicación de algún castigo si llegara a presentar cierta conducta inadecuada, con el

\section{Figura 1. Contingencias parentales ante conducta adecuada}

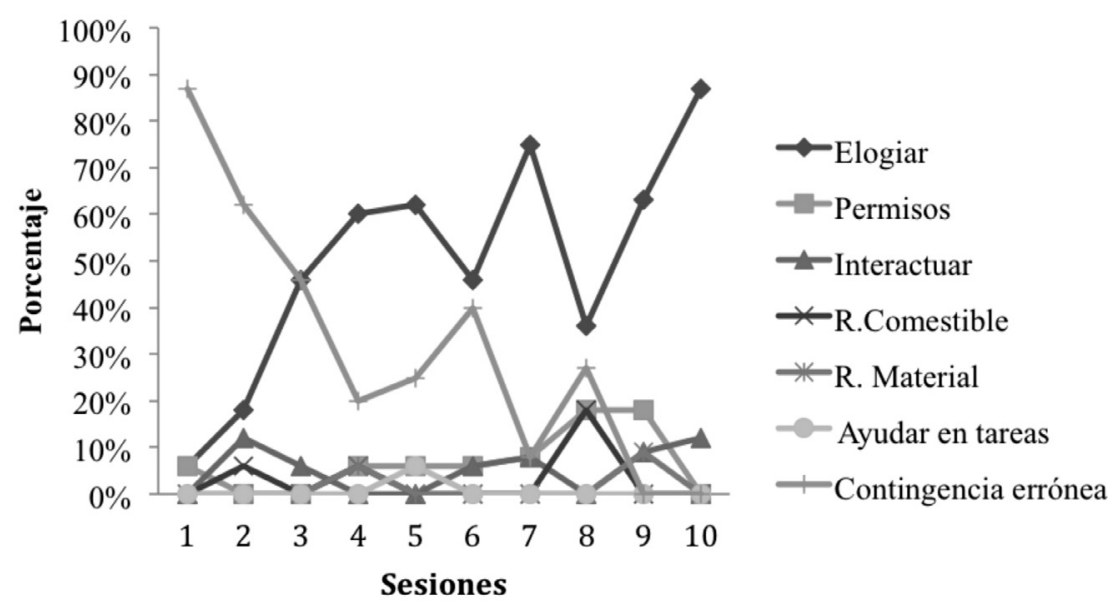


Figura 2. Contingencias parentales ante conducta inadecuada

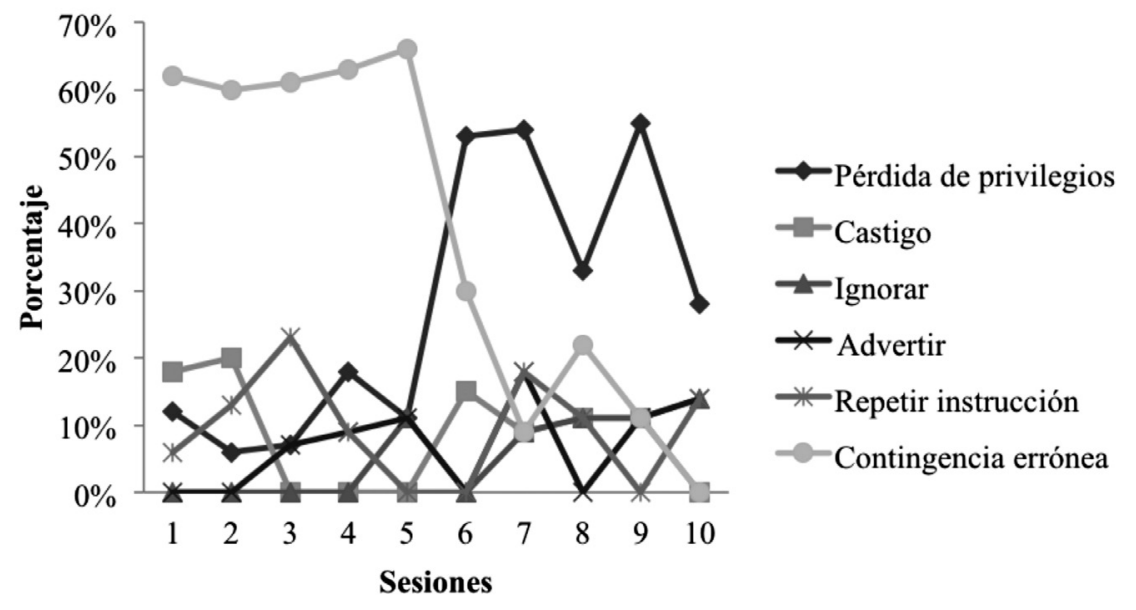

Figura 3. Modificación conductual promedio a lo largo de la intervención

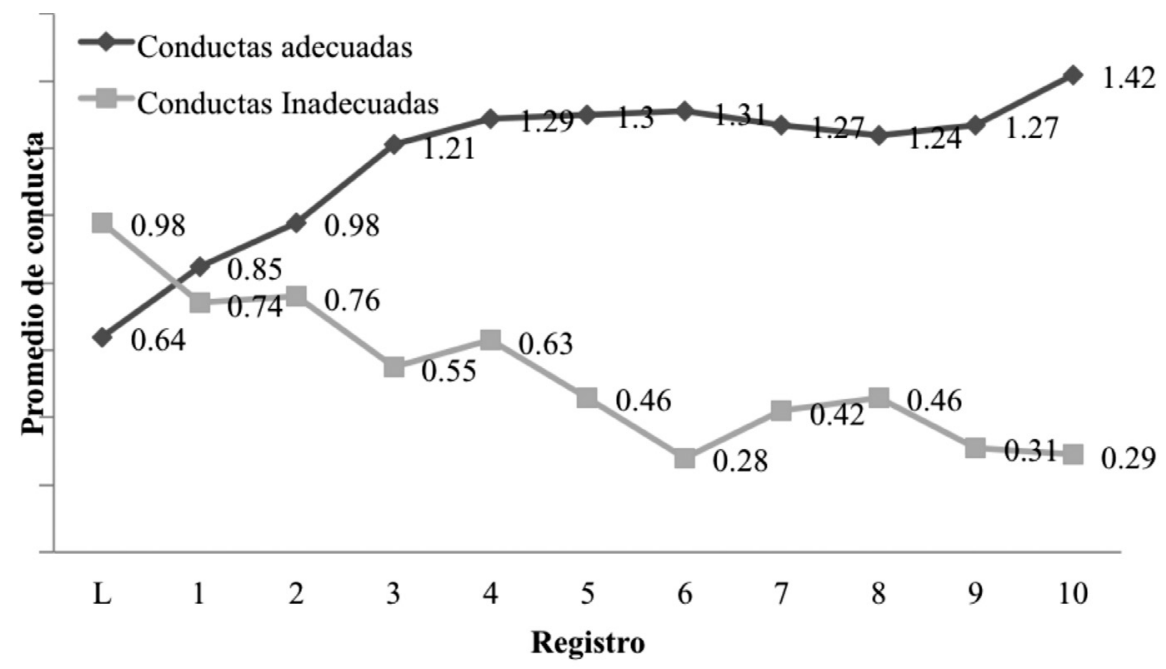

objetivo de frenarla sin necesidad de otorgar el castigo o la sanción; 5) repetir la instrucción dada al adolescente para que ejecute alguna conducta; y 6) contingencia errónea, al no presentar ninguna contingencia ante conducta inadecuada, como no decir nada, ceder, retirarse o dejar que la conducta continúe u otorgar alguna otra consecuencia positiva. Al inicio de la intervención esta última categoría fue la más frecuente pero se redujo y eliminó al final. Se observó lo contrario con la estrategia de pérdida de privilegios.

Cuando los padres aplicaron contingencias diferentes de las que solían aplicar, la conducta de sus hijos se vio modificada, incrementando la frecuencia de las conductas adecuadas y disminuyendo la de las inadecuadas (véase figura 3 ).

La percepción de problemas consecuentes de las conductas de sus hijos también se modificó. En la tabla 4 se presentan los porcentajes de frecuencias en las conductas problemáticas antes y después de la intervención. Al finalizar la intervención, los padres consideraron los problemas con sus hijos como muy leves (64\%), leves (27.3\%) e indeciso (9\%). La frecuencia percibida de los problemas fue de una vez al mes (54.5\%), menos de una vez al mes (27.3\%) y dos a tres veces por semana $(9 \%)$. 
Tabla 4. Percepción de problemáticas de los padres pre y postratamiento

\begin{tabular}{l|c|c}
\hline Conductas problemáticas & Pretratamiento & Postratamiento \\
\hline Consumo en sus hijos & $\%$ & $\%$ \\
\hline Problemas con otros miembros de la familia & 41 & 9 \\
\hline Con la pareja & 61 & 18 \\
\hline En la realización de proyectos de vida & 55 & 18 \\
\hline En la resolución de problemas propios & 55 & 9 \\
\hline Malestar emocional & 90 & 18 \\
\hline Frustración & 84 & 0 \\
\hline Incertidumbre & 93.5 & 9 \\
\hline Desmotivación & 80 & 0 \\
\hline Sentimiento de culpa & 87 & 9 \\
\hline
\end{tabular}

Tabla 5. Percepción de problemáticas de los adolescentes pre y postratamiento

\begin{tabular}{l|c|c}
\hline Problemáticas & Pretratamiento & Postratamiento \\
\hline Discusiones & $\%$ & $\%$ \\
\hline Sentirse rechazado/despreciado & 70.6 & 20 \\
\hline Desconfianza & 41 & 0 \\
\hline Problemas de comunicación & 59 & 0 \\
\hline Problemas de conducta en la escuela & 47 & 0 \\
\hline Desmotivación & 41 & 10 \\
\hline Frustración & 41 & 10 \\
\hline Confusión & 59 & 20 \\
\hline Malestar emocional & 53 & 0 \\
\hline
\end{tabular}

En el caso de los adolescentes, la evaluación inicial fue completada por un total de 30 participantes. La edad promedio fue de 14 años, $54 \%$ de ellos fueron mujeres. El 87.5\% refirió problemas con alguno de sus padres o con ambos; $35 \%$ habló de un conflicto grave en los últimos 12 meses. El 78\% refirió haber consumido alguna sustancia adictiva; $35 \%$ percibió diario la frecuencia de los problemas, de dos a tres veces por semana $29 \%$, una vez por semana $17.6 \%$, una vez por mes $11.8 \%$ y una vez cada 15 días $6 \%$. Los resultados de los hijos de los padres que concluyeron la intervención (17 participantes) muestran que las consecuencias negativas resultado de los problemas con los padres se modificaron tras la intervención (ver tabla 5). 


\section{DISCUSIÓN}

El programa aplicado para la reducción de conductas inadecuadas en adolescentes ha mostrado que las técnicas conductuales pueden ser efectivas a corto plazo para generar cambios notables, y que dichos cambios se deben a la correcta aplicación de los principios del condicionamiento operante (Kazdin, 2000). Estos hallazgos son consistentes con reportes de efectividad de otros estudios similares (Conger \& Simons, 1997; Kolivas, Riordan \& Gross, 2008), por lo que al generar estrategias de control conductual con adolescentes, es necesario tomar en cuenta estas técnicas, debido a su efectividad y a su aplicación práctica y sencilla que se puede enseñar a padres de familia independientemente de su escolaridad, como se demostró en este estudio.

Al disminuir conductas inadecuadas cotidianas, también se observó la no aparición de otras conductas más severas, lo cual se constató durante el tiempo que duró la intervención. Por ello, se puede decir que al mantener controlados problemas conductuales cotidianos, se pueden mantener contenidas problemáticas mayores, lo cual corresponde con los modelos de riesgo (Burt et al., 1998; Capuzzi \& Gross, 2004; Dryfoos, 1990).

Fue posible conocer de manera clara y objetiva cuáles eran las principales molestias de conducta entre padres e hijos, y también cómo les afectaban, aportando datos importantes respecto a las características de los problemas con los que conviven día a día, lo cual es relevante para la comprensión de problemas complejos, como por ejemplo, el desarrollo de conductas adictivas

\section{REFERENCIAS}

Bojórquez, I., Fernández-Varela, H., Gorab, A. \& Solís, C. (2010). Factors associated with illegal substance use initiation among young students in Mexico City. Drug and Alcohol Review, 29, 286-292.

Burt, M., Resnick, G. \& Novick, E. (1998). Building supportive communities for at-risk adolescents. Washington DC: American Psychological Association.

Capuzzi, D. \& Gross, D. (2004). Youth at Risk. EU: Pearson Merrill Prentice Hall.

Conger, R. D. \& Simons, R. L. (1997). Life-course contingencies in the development of adolescent antiso-
(NIDA, 2005). Al reducir la frecuencia de problemas conductuales, se redujo la percepción de consecuencias negativas tanto en padres como en adolescentes y la frecuencia percibida de problemas entre ambos, por lo que se muestra que la relación padre-hijo se vio beneficiada. Este beneficio puede contribuir al fortalecimiento de factores de protección familiares, que son pieza clave para la reducción de problemas de salud mayores (NIDA, 2005; Rosovsky, 2003; Villatoro et al., 2007).

Sin embargo, la intervención no estuvo libre de variables a las que no se pudo acceder, por ejemplo, relaciones complicadas en la dinámica familiar, falta de cooperación o incluso, sabotaje intencional por parte de algún miembro de la familia, variables socioeconómicas que no permiten recurrir a técnicas de pérdida de privilegios, la propia salud mental de los padres de familia, problemas de pareja, o casos de adolescentes con conductas ya en otra fase que no corresponde a la prevención sino al tratamiento, e incluso a la rehabilitación.

Otros detalles de la intervención pueden ser rediseñados para funcionar más eficazmente, como la tarea de llenado del registro conductual, integrar factores cognitivos que impidan la ejecución de las técnicas y la inclusión del adolescente de forma más activa en el programa. Es necesario revisar cómo funciona la intervención a largo plazo y hacerlo con una muestra más amplia para determinar si el aprendizaje se mantiene con el tiempo, así como para ver si efectivamente se han logrado frenar problemas conductuales que impliquen un mayor riesgo a la integridad física y/o mental de los adolescentes.

cial behavior: A Matching Law approach. En T. P. Thornberry (Ed.) Developmental theories of crime and delinquency. Advances in criminological theory (pp. 55-99). Piscataway: Transaction Publishers.

Cooper, J., Heron, T. \& Heward, W. (1987). Applied Behavior Analysis. Ohio: Merril Publishing Company. Corsi, E., Barrera, P., Flores, C. Perivanicich, X., \& Guerra C. (2009). Efectos de un programa combinado de técnicas de modificación conductual para la disminución de la conducta disruptiva y el aumento de la conducta prosocial en escolares chilenos. Acta Colombiana de Psicología, 12(1), 67-76. 
Diccionario enciclopédico (2009), vol. 1. México: Editorial Larousse.

Diccionario Manual de la lengua española (2007). (17a. ed., 2a. reimp.). México: Vox.

Dryfoos, I. C. (1990). Adolescents at risk; prevalence and prevention. NY: Oxford University Press.

Fulgencio, M. (2002). Entrenamiento en habilidades de comunicación y negociación para padres y adolescentes que presentan comportamiento agresivo. (Tesis de Maestría). Universidad Nacional Autónoma de México, Facultad de Psicología, México.

Fondo de las Naciones Unidas para la Infancia (2006). Informe nacional sobre violencia y salud. México: Secretaría de Salud.

Godley, S. H., Hedges, K., \& Hunter, B. (2011). Gender and racial differences in treatment process and outcome among participants in the adolescent community reinforcement approach. Psychology of Addictive Behaviors, 25(1), 143-154.

Jessor, R. (1992). Risk behavior in adolescence: a psychosocial framework for understanding and action. Developmental Review, 12, 374-390.

Kazdin, A. (2000). Modificación de la conducta y sus aplicaciones prácticas. México: El Manual Moderno.

Kolivas, E., Riordan, P. \& Gross, A. M. (2008). Overview of behavioral treatment with children and adolescents. En M. Hersen \& D. Reitman (Eds.) Handbook of psychological assessment, case conceptualization, and treatment, Vol 2: Children and adolescents (pp. 102-125). Hoboken: John Wiley \& Sons.

Learner, M. \& Spanier, B. (1978). A Dynamic International View of child and family development. En R. M. Learner \& G. B. Spanier (Eds.), Child Influences on marital and family. International life span perspective (pp. 174-181). NY: Academic.

Mahecha, J. (2006). Programa de prevención de la conducta agresiva y promoción de la conducta prosocial en niños y jóvenes. (Tesis de maestría). Fundación Universitaria Konrad Lorenz. Bogotá.
Marini, V. A. \& Stickle, T. R. (2010). Evidence for deficits in reward responsivity in antisocial youth with callous-unemotional traits. Personality Disorders: Theory, Research, and Treatment, 1(4), 218-229.

Millstein, S. G., Irwin, C. E., Adler, N. E., Cohn, 1. D., Kegeles, S. M. \& Dolcini, M. M. (1992). Health- Risk behaviors and health concerns among young adolescents. Pediatrics, 89, 422-428.

Morales, S., Martínez, M. \& Vázquez, F. (2007). Módulo de orientación para manejo de problemas en la infancia y la promoción de la salud familiar. México: Conadic.

National Institute of Drug Addiction (2005). What are risk factors and protective factors? Recuperado de www. drugabuse.gov/infofacts/, el 23 de febrero de 2011.

Neale, M. A. \& Bazerman, M. H. (1991). Cognition and rationality in negotiation. NY: Free Press.

Papalia, D., Wendkos, S. \& Duskin R. (2007). Psicología del desarrollo. México: McGraw-Hill Interamericana.

Patterson, G. (1975). Aprenda a convivir en familia. México: Ciencia de la Conducta.

Rebolledo, E., Medina, N. \& Pillon, S. (2004). Factores asociados al uso de drogas en estudiantes adolescentes. Revista Latino-am Emfermagem, 12 (especial), 369-375.

Rosendahl, K. I., Galanti, M. R. \& Gilljam, H. (2008). Trajectories of smokeless tobacco use and of cigarette smoking in a cohort of Swedish adolescents: Differences and implications. Nicotine \& Tobacco Research, 10(6), 1021-1027.

Rosovsky, H. (2003). El papel de la familia en la prevención y manejo del abuso del alcohol: una revisión de evidencias, Cuadernos FISAC, 1(17), 9-19.

Villatoro, J., Gutiérrez, M., Quiroz, N., Moreno, M., Gaytán, L., Gaytán, F., Amador, N. y Medina-Mora, M. E. (2007). Encuesta de Consumo de Drogas en Estudiantes 2006. México: Instituto Nacional de Psiquiatría Ramón de la Fuente Muñiz. 